\title{
A RESULT ON GENERALIZED DERIVATIONS WITH ENGEL CONDITIONS ON ONE-SIDED IDEALS
}

\author{
ÇaĞRi Demir and Nurcan ArgaÇ
}

\begin{abstract}
Let $R$ be a non-commutative prime ring and $I$ a non-zero left ideal of $R$. Let $U$ be the left Utumi quotient ring of $R$ and $C$ be the center of $U$ and $k, m, n, r$ fixed positive integers. If there exists a generalized derivation $g$ of $R$ such that $\left[g\left(x^{m}\right) x^{n}, x^{r}\right]_{k}=0$ for all $x \in I$, then there exists $a \in U$ such that $g(x)=x a$ for all $x \in R$ except when $R \cong M_{2}(G F(2))$ and $I[I, I]=0$.
\end{abstract}

\section{Introduction}

Throughout this paper unless specially stated, $R$ always denotes a prime ring with center $Z(R)$, extended centroid $C$, left Utumi quotient ring $U$, and two sided Martindale quotient ring $Q$. For any $x, y \in R$, we set $[x, y]_{1}=[x, y]=$ $x y-y x$ and $[x, y]_{k}=\left[[x, y]_{k-1}, y\right]$ for $k>1$.

We mean by a derivation of $R$ an additive mapping $d$ from $R$ into itself which satisfies the rule $d(x y)=d(x) y+x d(y)$ for all $x, y \in R$. A well-known result proved by Posner [21] states that $R$ must be commutative if there exists a nonzero derivation $d$ of $R$ such that $[d(x), x]=0$ for all $x \in R$. Many related generalizations have been obtained by a number of authors in the literature (e.g., see, [10], [14], [15], [16]).

An additive mapping $g: R \rightarrow R$ is called a generalized derivation of $R$ if there exists a derivation $d$ of $R$ such that $g(x y)=g(x) y+x d(y)$ for all $x, y \in R[9]$. Obviously any derivation is a generalized derivation. Moreover, another basic example of a generalized derivation is the mapping of the form $g(x)=a x+x b$ for $a, b \in R$. Many authors have studied generalized derivations in the context of prime and semiprime rings (see [1], [2], [3], [13], [9], [18]).

In [13], T. K. Lee extended the definition of a generalized derivation as follows. By a generalized derivation he means an additive mapping $g: J \rightarrow U$ such that $g(x y)=g(x) y+x d(y)$ for all $x, y \in J$, where $U$ is the right Utumi

Received July 3, 2008.

2000 Mathematics Subject Classification. 16N60, 16W25, $16 \mathrm{U} 80$.

Key words and phrases. prime rings, derivations, generalized derivations, left Utumi quotient rings, two-sided Martindale quotient ring, differential identities, Engel condition.

This research has been supported by The Scientific and Technological Research Council of Turkey, TUBITAK, No: 108T257. 
quotient ring of $R, J$ is a dense right ideal of $R$ and $d$ is a derivation from $J$ to $U$. He also proved that every generalized derivation can be uniquely extended to a generalized derivation of $U$. In fact, there exists $a \in U$ and a derivation $d$ of $U$ such that $g(x)=a x+d(x)$ for all $x \in U$ [13, Theorem 3]. A corresponding form to dense left ideals as follows. Let $I$ be a dense left ideal of $R$ and $U$ be the left Utumi quotient ring of $R$. An additive mapping $g: I \rightarrow U$ is called a generalized derivation if there exists a derivation $d: I \rightarrow U$ such that $g(x y)=x g(y)+d(x) y$ for all $x, y \in I$. Following the same methods in [13], one can extend $g$ uniquely to a generalized derivation of $U$, which we will also denote by $g$, and $g$ assumes the form $g(x)=x a+d(x)$ for all $x \in U$ and some $a \in U$, where $d$ is a derivation of $U$. Notice that $g(x)=a x+\left(d-d_{a}\right)(x)$ for all $x \in U$, where $d_{a}$ denotes the inner derivation induced by the element $a \in U$, i.e., $d_{a}(x)=[a, x]$. Setting $\delta=d-d_{a}$, we may always assume that a generalized derivation of a prime ring is of the form $g(x)=a x+\delta(x)$ for all $x \in U$, where $a \in U$ and $\delta$ is a derivation of $U$.

In [11], C. Lanski proved that if $R$ is a prime ring with derivation $d, I$ is a left ideal of $R$, and $k, n$ are positive integers such that $\left[d\left(x^{k}\right), x^{k}\right]_{n}=0$ for all $x \in I$, then either $d=0$ or $R$ is commutative. In [1], this result extended to generalized derivations.

In [17], T. K. Lee and W. K. Shiue showed that if $R$ is a non-commutative prime ring, $I$ is a nonzero left ideal of $R$ and $d$ is a derivation of $R$ such that $\left[d\left(x^{m}\right) x^{n}, x^{r}\right]_{k}=0$ for all $x \in I$, where $k, m, n, r$ are fixed positive integers, then $d=0$ except when $R \cong M_{2}(G F(2))$.

The aim of the present paper is to extend this result to generalized derivations. Precisely, we will prove the following.

Theorem 1. Let $R$ be a non-commutative prime ring and $k, m, n, r$ fixed positive integers. If there exists a generalized derivation $g$ of $R$ such that $\left[g\left(x^{m}\right) x^{n}, x^{r}\right]_{k}=0$ for all $x \in R$, then there exists an element $a \in U$ such that $g(x)=x a$ for all $x \in R$.

Theorem 2. Let $R$ be a non-commutative prime ring, I a non-zero left ideal of $R$ and $k, m, n, r$ fixed positive integers. If there exists a generalized derivation $g$ of $R$ such that $\left[g\left(x^{m}\right) x^{n}, x^{r}\right]_{k}=0$ for all $x \in I$, then there exists $a \in U$ such that $g(x)=x a$ for all $x \in R$ except when $R \cong M_{2}(G F(2))$ and $I[I, I]=0$.

\section{Preliminaries}

In what follows, unless stated otherwise, $R$ will be a prime ring. The related object we need to mention is the left Utumi quotient ring $U$ of $R$ (sometimes, as in [4], $U$ is called the maximal left ring of quotients).

The definitions, the axiomatic formulations and the properties of this quotient ring $U$ can be found in [4].

In any case, when $R$ is a prime ring, all we need about $U$ is that

1) $R \subseteq U$;

2) $U$ is a prime ring; 
3) The center of $U$, denoted by $C$, is a field which is called the extended centroid of $R$.

We also frequently use the theory of generalized polynomial identities and differential identities (see [4], [10], [12], [20]). In particular we need to recall the following:

Remark 1 ([6]). If $R$ is a prime ring and $I$ is a non-zero left ideal of $R$, then $I, R I$ and $U I$ satisfy the same generalized polynomial identities.

Remark 2 ([10]). Let $R$ be a prime ring, $d$ a nonzero derivation of $R$ and $I$ a nonzero two-sided ideal of $R$. Let $f\left(x_{1}, \ldots, x_{n}, d\left(x_{1}\right), \ldots, d\left(x_{n}\right)\right)$ be a differential identity in $I$, that is

$$
f\left(r_{1}, \ldots, r_{n}, d\left(r_{1}\right), \ldots, d\left(r_{n}\right)\right)=0 \text { for all } r_{1}, \ldots, r_{n} \in I .
$$

Then one of the following holds:

1) Either $d$ is an inner derivation in $Q$, the Martindale quotient ring of $R$, in the sense that there exists $q \in Q$ such that $d(x)=[q, x]$ for all $x \in R$, and $I$ satisfies the generalized polynomial identity

or

$$
f\left(r_{1}, \ldots, r_{n},\left[q, r_{1}\right], \ldots,\left[q, r_{n}\right]\right)
$$

2) $I$ satisfies the generalized polynomial identity

$$
f\left(x_{1}, \ldots, x_{n}, y_{1}, \ldots, y_{n}\right) .
$$

\section{Results}

We need the following lemmas.

Lemma 1. Let $R=M_{t}(F)$, where $F$ is a field, $t \geq 2$ and $a, b \in R$. Suppose that

$$
\left[a x^{m+n}+\left[b, x^{m}\right] x^{n}, x^{r}\right]_{k}=0 \quad \text { for all } x \in R,
$$

where $k, m, n, r$ are fixed positive integers. Then $a+b \in F$.

Proof. Let $e$ be an idempotent element in $R$. Setting $x=e$ in (1) and multiplying left side by $(1-e)$, we see that $(1-e)(a+b) e=0$ for any idempotent element $e$. Thus $a+b$ is a diagonal matrix. Note that $u(a+b) u^{-1}$ must be diagonal for each invertible element $u \in R$, since

$$
\left[\left(u a u^{-1}\right) x^{m+n}+\left[\left(u b u^{-1}\right), x^{m}\right] x^{n}, x^{r}\right]_{k}=0
$$

for all $x \in R$. Write $a+b=\sum_{i=1}^{t} \beta_{i} e_{i i}$, where $\beta_{i} \in F$. Then for each $j>1$, we see that $\beta_{j}-\beta_{1}$, the $(1, j)$-entry of $\left(1+e_{1 j}\right)(a+b)\left(1+e_{1 j}\right)^{-1}$, equals 0 . That is, $\beta_{j}=\beta_{1}$ for $j>1$ and hence $a+b \in F$.

Lemma 2. Let $R$ be a non-commutative prime ring and $a, b \in R$ such that $\left[a x^{m+n}+\left[b, x^{m}\right] x^{n}, x^{r}\right]_{k}=0$ for all $x \in R$, where $k, m, n, r$ are fixed positive integers. Then $a+b \in Z(R)$. 
Proof. Suppose on the contrary that $a+b \notin C$. Then

$$
f(X)=\left[(a+b) X^{m+n}-X^{m} b X^{n}, X^{r}\right]_{k}
$$

is a nontrivial generalized polynomial identity (GPI) for $R$. By [6], $f(X)$ is also a GPI for $Q$. Denote by $F$ either the algebraic closure of $C$ or $C$ according to the cases where $C$ is either infinite or finite, respectively. Then, by a standard argument (e.g., see [19, Proposition]), $f(X)$ is also a $G P I$ for $Q \otimes_{C} F$. Since $Q \otimes_{C} F$ is centrally closed prime $F$-algebra [7, Theorems 2.5 and 3.5], by replacing $R, C$ with $Q \otimes_{C} F, F$, respectively we may assume $R$ is centrally closed and $C$ is either finite or algebraically closed. In view of Martindale's theorem [20], $R$ is a primitive ring having a non-zero socle $H$ with $C$ as its associated division ring.

Since $a+b \notin C$, we have $[a+b, h] \neq 0$ for some $h \in H$. By Litoff's theorem [8], there exists an idempotent $e \in H$ such that $h, a h, h a, h b, b h \in e R e$. Note that $e f(e X e) e$ is a $G P I$ for $R$. Thus, $\left[(e a e) X^{m+n}+\left[e b e, X^{m}\right] X^{n}, X^{r}\right]_{k}$ is a GPI for $e R e$. Since $e R e \cong M_{s}(C)$ for some $s \geq 1$ then eae $+e b e$ is central in $e R e$ by Lemma 1. Then there exists $c \in C$ such that $c e=e a e+e b e$. Hence $c h=e a e h+e b e h=e a h+e b h=a h+b h=(a+b) h$. Similarly $h c=h e a e+h e b e=$ $h a e+h b e=e h a e+e h b e=h a+h b=h(a+b)$. So $[a+b, h]=0$, a contradiction. Therefore $a+b \in Z(R)$.

Corollary 1. Let $R$ be a prime ring and $a \in R$ such that $\left[a x^{m}, x^{n}\right]_{k}=0$ for all $x \in R$, where $k, m, n$ are fixed positive integers. Then $a \in Z(R)$.

Proof of Theorem 1. As we have already noted that every generalized derivation $g$ on a dense left ideal of $R$ can be uniquely extended to $U$ and assumes the form $g(x)=a x+d(x)$ for some $a \in U$ and a derivation $d$ on $U$. If $d=0$, then $\left[a x^{m+n}, x^{r}\right]_{k}=0$ for all $x \in R$. By Remark 1, $U$ satisfies the above generalized identity. Moreover, since $U$ remains prime by the primeness of $R$, replacing $R$ with $U$, we may assume that $a \in R$ and $C$ is just the center of $R$. By Corollary 1 , we have $a \in Z(R)$. Thus $g(x)=a x=x a$ for all $x \in R$. So we may assume that $d \neq 0$.

In the light of Remark 2, we divide the proof into two cases:

Case 1. Let $d$ be the inner derivation induced by the element $b \in U-C$, that is, the $d(x)=[b, x]$ for all $x \in U$. Then $R$ satisfies the nontrivial generalized polynomial identity

$$
\left[a x^{m+n}+\left[b, x^{m}\right] x^{n}, x^{r}\right]_{k} .
$$

By Remark 1, $U$ satisfies the above generalized polynomial identity. Moreover, since $U$ remains prime by the primeness of $R$, replacing $R$ with $U$, we may assume that $a, b \in R$ and $C$ is just the center of $R$. Then by Lemma 2 , we have that $a+b \in Z(R)$. Therefore $g(x)=a x+[b, x]=(a+b) x-x b=x(a+b-b)=x a$ for all $x \in R$.

Case 2. Let now $d$ be an outer derivation of $U$. To continue the proof, we set $G(Y, X)=\sum_{i=0}^{m-1} X^{i} Y X^{m-1-i}$, a non-commuting polynomial in variables 
$X$ and $Y$. Note that $d\left(x^{m}\right)=G(d(x), x)$. Then $R$ satisfies the following differential identity

$$
\left[a x^{m+n}+G(d(x), x) x^{n}, x^{r}\right]_{k} .
$$

Now by Remark 2, $R$ satisfies the identity

$$
\left[a x^{m+n}+G(y, x) x^{n}, x^{r}\right]_{k} .
$$

Taking $y=0$ in the above identity, we get

$$
\left[a x^{m+n}, x^{r}\right]_{k}=0 \text { for all } x \in R \text {. }
$$

So we have

$$
\left[d\left(x^{m}\right) x^{n}, x^{r}\right]_{k}=0 \text { for all } x \in R .
$$

Therefore by $[17$, Theorem 1$]$, we must have $d=0$, a contradiction. This proves the result.

By using almost the same argument in [17], we have the following.

Lemma 3. Let $R=M_{l}(F)$, where $F$ is a field, $l \geq 2$, and $I$ a minimal left ideal of $R$. Suppose $\left[a x^{m+n}+\left[b, x^{m}\right] x^{n}, x^{r}\right]_{k}=0$ for all $x \in I$, where $m, n, r, k$ are fixed positive integers. Then $a+b \in F$ except when $R \cong M_{2}(G F(2))$.

Proof. Suppose that $a+b \notin F$. Since $I$ is a minimal left ideal, it is clear that we may assume $I=R e_{11}$. Let $e=e^{2} \in I$. By the hypothesis, we have $[a e+[b, e] e, e]_{k}=0$. Left multiplying by $1-e$, we see that

$$
(1-e)(a+b) e=0 \text { for all } e \in I \text {. }
$$

Let $\beta \in F$ and $x \in R$. Then $f=e+(1-e) x e$ and $g=e+\beta(1-e) x e$ are idempotents in $I$. Set $c=a+b$, then $c \notin F$ and $(1-e) c e=0$. Thus by (2) we have $(1-f) c f=0=(1-g) c g$. Therefore we see that

$$
((1-e)-(1-e) x e) c(e+(1-e) x e)=0
$$

and

$$
((1-e)-\beta(1-e) x e) c(e+\beta(1-e) x e)=0 .
$$

Using (2) we arrive at the following equations:

$$
(1-e) c x e-(1-e) x c e-(1-e) x e c(1-e) x e=0
$$

and

$$
\beta(1-e) c x e-\beta(1-e) x c e-\beta^{2}(1-e) x e c(1-e) x e=0 .
$$

Multiplying first equation by $\beta$ and comparing the last two equations we see that $\left(\beta^{2}-\beta\right)(1-e) x e c(1-e) x e=0$ for all $x \in R$. Then either $\beta \in\{0,1\}$ or $e c(1-e)=0$ for any idempotent $e \in I$. Suppose that the second possibility holds. In particular, $e_{11} c\left(1-e_{11}\right)=0$. Let $x \in R$. Then we have $x e_{11} c=$ $x e_{11} c e_{11}=\mu x e_{11}$ for some $\mu \in F$. Thus we see that $I(c-\mu)=\operatorname{Re}_{11}(c-\mu)=0$ for some $\mu \in F$. On the other hand, in view of (2) we get $[c, e]=0$ for any idempotent $e \in I$. Then $0=[c, e]=[c-\mu, e]=(c-\mu) e$ for all idempotent 
$e \in I$. Note that $e_{11}+\left(1-e_{11}\right) x e_{11} \in I$ is also an idempotent for every $x \in R$. Hence $(c-\mu)\left(e_{11}+\left(1-e_{11}\right) x e_{11}\right)=0$ for all $x \in R$. Since $(c-\mu) e_{11}=0$, it is clear that $(c-\mu) R e_{11}=0$. Therefore $c=\mu \in F$, a contradiction. Thus we get $F=G F(2)$.

Now we prove that $l=2$. Suppose on the contrary that $l>2$. Let $i, j$ be two distinct positive integers such that $2 \leq i, j \leq l$. Then $e_{11}, e_{11}+e_{i 1}, e_{11}+e_{j 1}$ and $e_{11}+e_{i 1}+e_{j 1}$ are idempotents in $I$. In view of (2) we obtain that

$$
\begin{gathered}
c e_{11}=e_{11} c e_{11}, \\
c\left(e_{11}+e_{i 1}\right)=\left(e_{11}+e_{i 1}\right) c\left(e_{11}+e_{i 1}\right), \\
c\left(e_{11}+e_{j 1}\right)=\left(e_{11}+e_{j 1}\right) c\left(e_{11}+e_{j 1}\right)
\end{gathered}
$$

and

$$
c\left(e_{11}+e_{i 1}+e_{j 1}\right)=\left(e_{11}+e_{i 1}+e_{j 1}\right) c\left(e_{11}+e_{i 1}+e_{j 1}\right) .
$$

Using $c e_{11}=e_{11} c e_{11}$ and comparing the other equations in (3), we arrive at $e_{i 1} c e_{j 1}+e_{j 1} c e_{i 1}=0$. Set $c=\sum_{1 \leq i, j \leq l} \beta_{i j} e_{i j}$, where $\beta_{i j} \in F$. Then this implies that $\beta_{1 j}=0=\beta_{1 i}$. Hence the second equation in (3) reduces to $c e_{i 1}=\beta_{11} e_{i 1}$, and so $\beta_{p i}=0$ for $p \neq i$ and $\beta_{i i}=\beta_{11}$. Thus we get $c=a+b \in F$, a contradiction. This proves the lemma.

Lemma 4. Let $R$ be a prime ring, $I$ a non-zero left ideal of $R$ and $a \in R$ such that $\left[a x^{m}, x^{n}\right]_{k}=0$ for all $x \in I$, where $k, m, n$ are fixed positive integers. Then $a \in Z(R)$ except when $R \cong M_{2}(G F(2))$ and $I[I, I]=0$.

Proof. Assume that $\left[a x^{m}, x^{n}\right]_{k}=0$ for all $x \in I$. Then

$$
\left[\left[a, x^{n}\right] x^{m}, x^{n}\right]_{k}=\left[a x^{m}, x^{n}\right]_{k+1}=0
$$

for all $x \in I$. Now by [17, Lemma 3] we have $a \in Z(R)$ except when $R \cong$ $M_{2}(G F(2))$ and $I[I, I]=0$.

Lemma 5. Let $R$ be a non-commutative prime ring and $I$ a non-zero left ideal and $a, b \in R$ such that

$$
\left[a x^{m+n}+\left[b, x^{m}\right] x^{n}, x^{r}\right]_{k}=0 \quad \text { for all } x \in I,
$$

where $k, m, n, r$ are fixed positive integers. Then $a+b \in Z(R)$ except when $R \cong M_{2}(G F(2))$ and $I[I, I]=0$.

Proof. Assume that $a+b \notin C$. If $I(b-\beta)=0$ for some $\beta \in C$, then setting $b^{\prime}=b-\beta$ we have $I b^{\prime}=0$. Moreover by (4) it is clear that

$$
\left[a x^{m+n}+\left[b^{\prime}, x^{m}\right] x^{n}, x^{r}\right]_{k}=0 \quad \text { for all } x \in I \text {. }
$$

Thus we get

$$
\left[\left(a+b^{\prime}\right) x^{m+n}, x^{r}\right]_{k}=0 \text { for all } x \in I .
$$

By Remark 1, $\left[\left(a+b^{\prime}\right) x^{m+n}, x^{r}\right]_{k}=0$ for all $x \in U I$. Moreover $U I b^{\prime}=0$ if and only if $I b^{\prime}=0$. Now $I$ and $U I$ satisfy the same basic conditions. Hence 
replacing $R, I$ with $U, U I$, respectively, we may assume that $a, b^{\prime} \in R$ and $C$ is just the center of $R$. Thus we get the conclusion $R \cong M_{2}(G F(2))$ and $I[I, I]=0$ since $a+b^{\prime} \notin C$.

So we may assume that $I(b-\beta) \neq 0$ for all $\beta \in C$. Hence, in view of $[14$, Lemma 3], either $R$ is a PI-ring or there exists an element $u \in I$ such that $u b$ and $u$ are $C$-independent. For the latter case,

$$
\left[a(X u)^{m+n}+\left[b,(X u)^{m}\right](X u)^{n},(X u)^{r}\right]_{k}
$$

is a non-trivial GPI for $R$.

On the other hand we have $\left[a x^{m+n}+\left[b, x^{m}\right] x^{n}, x^{r}\right]_{k}=0$ for all $x \in Q I$ by [6]. Thus applying the same argument in Lemma 2 and replacing $I$ by $Q I$, we may assume that $R$ is a centrally closed prime ring having a non-zero socle $H$, with $C$ as its associated division ring and $I=I C$. Moreover $C$ is either algebraically closed or finite. If $R$ contains no non-trivial idempotents, then $R$ is a division ring and $I=R$. Then by the proof of Theorem 1 we obtain that $a+b \in C$, a contradiction. So we may assume that $R$ contains a non-trivial idempotent. On the other hand we have

$-I[I, I]=0$ if and only if $H I[H I, H I]=0$ by $[6]$,

- $I(b-\mu)=0$ if and only if $H I(b-\mu)=0$ for some $\mu \in C$.

So replacing $I$ by $H I$ we may assume $I \subseteq H$. Suppose first that $I[I, I] \neq 0$. Then $I$ always contains an idempotent with rank 2 or greater that 2 . Let $e$ be such an idempotent in $I$.

Now choose $x$ as exe in (4), then

$$
\left[a(e x e)^{m+n}+\left[b,(e x e)^{m}\right](e x e)^{n},(e x e)^{r}\right]_{k}=0 \quad \text { for all } x \in R,
$$

and left-side multiplying by $e$ yields

$$
\left[(e a e)(e x e)^{m+n}+\left[e b e,(e x e)^{m}\right](e x e)^{n},(e x e)^{r}\right]_{k}=0 \quad \text { for all } x \in R .
$$

But $e R e \cong M_{l}(C)$, where $l=\operatorname{rank}(e) \geq 2$. By Lemma 2 we have $e(a+b) e \in C e$. Choosing $x=e$ in (4), we get $(a+b) e-e(a+b) e=0$. Then $a e+b e \in C e$ for every idempotent $e \in I$ with $\operatorname{rank}(e) \geq 2$. Note that $e+(1-e) x e \in I$ is also an idempotent for all $x \in R$ and $\operatorname{rank}(e+(1-e) x e)=\operatorname{rank}(e) \geq 2$. Set $c=a+b$, so $c e \in C e$ for all idempotent $e \in I$ with $\operatorname{rank}(e) \geq 2$. In particular we have

$$
c(e+(1-e) x e) \in C(e+(1-e) x e)
$$

for all $x \in R$. Left-side multiplying by $e$ yields that

$$
\text { ece }+ \text { ecxe }- \text { ecexe } \in \text { Ce. }
$$

Since $e c e=c e \in C e$, we get $[e, c] x e \in C e$ for all $x \in R$. Suppose for the moment that $[e, c] \neq 0$. Choose $x_{0} \in R$ such that $[e, c] x_{0} e=\beta e \neq 0$ for some $\beta \in C$. Then we have $\beta$ exe $=[e, c] x_{0}$ exe $\in C e$. Therefore $e R e=C e$, because $\beta \neq 0$. But $e R e=C e$ implies that $\operatorname{rank}(e)=1$, a contradiction. Hence $[e, c]=0$. Now since $I$ is completely reducible left $H$-module, each element of $I$ is contained in $H f$ for some $f^{2}=f \in I$ with $\operatorname{rank}(f) \geq 2$. But $f c=c f \in C f$. 
Let $x \in I$. Then $x=h f$ for some $h \in H$. We see that $x c=h f c \in C h f=$ $C x$, and so $[x c, x]=0$ for all $x \in I$. Linearizing this last equation, we get

$$
[x c, y]+[y c, x]=0 \quad \text { for all } x, y \in I \text {. }
$$

Replacing $y=e$ in (6) and using the fact that $[e, c]=0$, we obtain

$$
0=[x c, e]+[e c, x]=e[c, x] \text { for all } x \in I .
$$

Hence we have $0=e[c, x y]=e x[c, y]$ for all $x, y \in I$. Therefore we get $e R I[c, I]=(0)$, and so $I[c, I]=0$. In particular, $[x[c, x], x]=0$ for all $x \in I$. So in view of $[17$, Lemma $3(\mathrm{ii})]$ one obtains $I(c-\lambda)=0$ for some $\lambda \in C$. Let $x \in R$, then it is clear that $f=e+(1-e) x e \in I$ is an idempotent with $\operatorname{rank}(f)=\operatorname{rank}(e) \geq 2$. Since $[c, e]=0$ for all $e=e^{2} \in I$ with $\operatorname{rank}(e) \geq 2$, in particular we have

$$
[c-\lambda, e+(1-e) x e]=0 \text { for all } x \in R .
$$

Hence we get $(c-\lambda) e+(c-\lambda)(1-e) x e=0$. On the other hand we have $(c-\lambda) e=[c-\lambda, e]=0$. So $(c-\lambda) x e=0$ for all $x \in R$. Thus the primeness of $R$ implies that $c=\lambda \in C$, and hence $a+b=c \in Z(R)$, a contradiction. This proves that $I[I, I]=0$.

If now $H \cong M_{l}(C)$ for some $l \geq 2$, then in view of Lemma 3, we are done. Thus we may assume $H \nsubseteq M_{l}(C)$ for all $l \geq 2$. Since $c \notin C$, it is clear that $c h \neq h c$ for some $h \in I$. It follows from Litoff's theorem [8] that there exists $e=e^{2} \in H, \operatorname{rank}(e) \geq 3$, such that $c h, h c, h \in e H e$. Note that ece $\notin C e$. Indeed, if ece $\in C e$, then eceh $=$ hece, and hence $c h=h c$, a contradiction. On the other hand, $0 \neq h \in I \cap e R e$. Since $R$ is centrally closed, $I C=I$ and $I[I, I]=0$, it is clear that $I$ is a minimal left ideal of $R$ by [5, Lemma 5.1]. We also have that $I \cap e R e$ is still a minimal left ideal of $e R e$ and $e R e \cong M_{l}(C)$, where $l=\operatorname{rank}(e) \geq 3$. Indeed, if $J$ is a left ideal of $e R e$ such that $J \varsubsetneqq I \cap e R e$, then $R J \varsubsetneqq R I \varsubsetneqq I$. Using the fact that $R J$ is a left ideal of $R$ such that $R J \varsubsetneqq I$ and $I$ is a minimal ideal of $R$, we get $R J=0$. Hence $J=0$ by the primeness of $R$. Now by the hypothesis, we have

$$
\left[(\text { eae })(\text { exe })^{m+n}+\left[\text { ebe, }(\text { exe })^{m}\right](\text { exe })^{n},(\text { exe })^{r}\right]_{k}=0 \quad \text { for all } x \in R,
$$

and so

$$
\left[(\text { eae }) x^{m+n}+\left[e b e, x^{m}\right] x^{n}, x^{r}\right]_{k}=0 \quad \text { for all } x \in I \cap e R e .
$$

In view of Lemma 3 this yields that $e R e \cong M_{2}(G F(2))$, a contradiction. This proves the result.

Example 1. Let $R=M_{s}(F), s>1$, the $s \times s$ matrices over a field $F$ and $I=R e_{11}$. If we set $a=1-e_{s 1}$ and $b=e_{s 1}$, then $\left[a x^{m+n}+\left[b, x^{m}\right] x^{n}, x^{r}\right]_{k}=0$ for all $x \in I$, where $k, m, n, r$ are fixed positive integers and $a+b \in Z(R)$.

Proof of Theorem 2. As we have already noted that every generalized derivation $g$ on a dense left ideal of $R$ can be uniquely extended to $U$, we may assume that $g$ has the form $g(x)=a x+d(x)$ for some $a \in U$ and a derivation $d$ on $U$. If $d=0$, then $\left[a x^{m+n}, x^{r}\right]_{k}=0$ for all $x \in I$. Then by Lemma 4 we have $a \in C$ 
except when $R \cong M_{2}(G F(2))$ and $I[I, I]=0$. If $a \in C$, then $g(x)=a x=x a$ for all $x \in R$. So we may assume that $d \neq 0$.

In the light of Remark 2, we divide the proof into two cases:

Case 1. Let $d$ be the inner derivation induced by the element $b \in U-C$, that is, $d(x)=[b, x]$ for all $x \in U$. Then $I$ satisfies the nontrivial generalized polynomial identity

$$
\left[a X^{m+n}+\left[b, X^{m}\right] X^{n}, X^{r}\right]_{k} .
$$

By Remark 1, $R I$ satisfies the above generalized identity. Since by [4], $R$ and $U$ satisfy the same GPIs, we have that $U I$ satisfies above identity. Then applying Lemma 5 to $U I$, we have that $a+b \in C$ except when $U \cong M_{2}(G F(2))$ and $U I[U I, U I]=0$. Moreover as in the proof of Lemma 5 we may replace $R, I$ by $U, U I$, respectively. Then in particular, $a+b \in C$ except when $R \cong M_{2}(G F(2))$ and $I[I, I]=0$. If $a+b \in C$, then $g(x)=a x+[b, x]=(a+b) x-x b=$ $x(a+b-b)=x a$ for all $x \in R$.

Case 2. Let now $d$ be an outer derivation of $U$. To continue the proof we set $G(Y, X)=\sum_{i=0}^{m-1} X^{i} Y X^{m-1-i}$, a non-commuting polynomial in variables $X$ and $Y$. Note that $d\left(x^{m}\right)=G(d(x), x)$. Since

$$
\left[a x^{m+n}+G(d(x), x) x^{n}, x^{r}\right]_{k} .
$$

is an identity for $I$, then for any $u \in I-C$

$$
\left[a(x u)^{m+n}+G(d(x u), x u)(x u)^{n},(x u)^{r}\right]_{k}
$$

is an identity for $R$. Thus $R$ satisfies the following

$$
\left[a(x u)^{m+n}+G(d(x) u+x d(u), x u)(x u)^{n},(x u)^{r}\right]_{k} .
$$

From Remark 2, since $d$ is an outer derivation $R$ satisfies the following identity

$$
\left[a(x u)^{m+n}+G(y u+x d(u), x u)(x u)^{n},(x u)^{r}\right]_{k} .
$$

Taking $y=0$ in (7) we get

$$
\left[a(x u)^{m+n}+G(x d(u), x u)(x u)^{n},(x u)^{r}\right]_{k}=0 .
$$

By the linearity of $g(Y, X)$ on $Y$, subtracting equation (7) from (8) yields that $R$ satisfies

$$
\left[G(y u, x u)(x u)^{n},(x u)^{r}\right]_{k}=0,
$$

which means that $R$ satisfies

$$
\begin{aligned}
0 & =\left[\sum_{i+j=m-1}(x u)^{i}(y u)(x u)^{j+n},(x u)^{r}\right]_{k} \\
& =\sum_{i+j=m-1}(x u)^{i}\left[(y u),(x u)^{r}\right]_{k}(x u)^{j+n} .
\end{aligned}
$$

Clearly (9) is a nontrivial GPI for $R$, since $u \notin C$. So $R C$ is a primitive ring with a non-zero socle $H([20]) . J=H I$ is a non-zero left ideal of $H$. Note that $H$ is simple, $J=H J$ and $J$ satisfies the same basic conditions as $I$ ([12]). Now 
replace $R$ by $H$ and $I$ by $J$, then, without loss of generality, $R$ is simple and equal to its own socle and $R I=I$. Let $e^{2}=e$ be some non-trivial idempotent in $I$. So for all $x, y \in R$, we have

$$
\sum_{i+j=m-1}(x e)^{i}\left[(y e),(x e)^{r}\right]_{k}(x e)^{j+n}=0
$$

and choosing $y=(1-e) r \in R$ we get

$$
(1-e)(r e)(x e)^{k r+m+n-1}=0 .
$$

This leads to the contradiction that either $e=0$ or $e=1$. Thus any idempotent element of $I$ is trivial, that is, $I=R$. Therefore we have to consider the condition

$$
\sum_{i+j=m-1} x^{i}\left[y, x^{r}\right]_{k} x^{j+n}=0
$$

for all $x, y \in R$, which is a polynomial identity. From Lemma 2 in [11], it follows that there exists a suitable field $F$ such that $R \subseteq M_{s}(F)$, the ring of all $s \times s$ matrices over $F$, and moreover $M_{s}(F)$ satisfies the same polynomial identity of $R$. In particular $M_{s}(F)$ satisfies

$$
\sum_{i+j=m-1} x^{i}\left[y, x^{r}\right]_{k} x^{j+n}=0 .
$$

Suppose $s \geq 2$ and choose $x=e_{11}$ and $y=e_{21}$ in (10). Then we have $e_{21}=0$. Thus $s=1$ and $R$ is commutative, a contradiction.

The following example shows our results do not hold in semiprime rings:

Example 2. Let $F$ be any field. Consider the semiprime ring

$$
R=\left(\begin{array}{ccc}
G F(2) & G F(2) & 0 \\
G F(2) & G F(2) & 0 \\
0 & 0 & F
\end{array}\right)
$$

Let

$$
I=\left(\begin{array}{ccc}
G F(2) & 0 & 0 \\
G F(2) & 0 & 0 \\
0 & 0 & F
\end{array}\right)
$$

be the left ideal of $R$. If $a=\left(\begin{array}{lll}1 & 1 & 0 \\ 0 & 0 & 0 \\ 0 & 0 & 1\end{array}\right)$ and $b=\left(\begin{array}{lll}0 & 0 & 0 \\ 0 & 0 & 0 \\ 0 & 0 & \alpha\end{array}\right)$ for $\alpha \in F$ fixed, one can easily see that $\left[a x^{2}+[b, x] x, x\right]=0$ for all $x \in I$, since $u v(u+v)=0$ for all $u, v \in G F(2)$. Then $g(x)=a x+[b, x]=(a+b) x-x b$ is a generalized derivation such that $[g(x) x, x]=0$ for all $x \in I$. But

$$
a+b \notin C=\left\{\left(\begin{array}{ccc}
\lambda & 0 & 0 \\
0 & \lambda & 0 \\
0 & 0 & \mu
\end{array}\right) \mid \lambda \in G F(2), \mu \in F\right\} .
$$




\section{References}

[1] E. Albaş, N. Argaç, and V. De Filippis, Generalized derivations with Engel conditions on one-sided ideals, Comm. Algebra 36 (2008), no. 6, 2063-2071.

[2] N. Argaç, L. Carini, and V. De Filippis, An Engel condition with generalized derivations on Lie ideals, Taiwanese J. Math. 12 (2008), no. 2, 419-433.

[3] N. Argaç, V. De Filippis, and H. G. Inceboz, Generalized derivations with power central values on multilinear poynomials on right ideals, to appear in Rendiconti di Padova,

[4] K. I. Beidar, W. S. Martindale III, and A. V. Mikhalev, Rings with Generalized Identities, Marcel Dekker, Inc., New York, 1996.

[5] M. Bresar, On generalized biderivations and related maps, J. Algebra 172 (1995), no. $3,764-786$.

[6] C. L. Chuang, GPIs having coefficients in Utumi quotient rings, Proc. Amer. Math. Soc. 103 (1988), no. 3, 723-728.

[7] J. S. Erickson, W. S. Martindale III, and J. M. Osborn, Prime nonassociative algebras, Pacific J. Math. 60 (1975), no. 1, 49-63.

[8] C. Faith and Y. Utumi, On a new proof of Litoff's theorem, Acta Math. Acad. Sci. Hungar 14 (1963), 369-371.

[9] B. Hvala, Generalized derivations in rings, Comm. Algebra 26 (1998), no. 4, 1147-1166.

[10] V. K. Kharchenko, Differential identities of prime rings, Algebra i Logika 17 (1978), no. 2, 220-238, 242-243.

[11] C. Lanski, An Engel condition with derivation for left ideals, Proc. Amer. Math. Soc. 125 (1997), no. 2, 339-345.

[12] T. K. Lee, Semiprime rings with differential identities, Bull. Inst. Math. Acad. Sinica 20 (1992), no. 1, 27-38.

[13] - Generalized derivations of left faithful rings, Comm. Algebra 27 (1999), no. 8, 4057-4073.

[14] Left annihilators characterized by GPIs, Trans. Amer. Math. Soc. 347 (1995), no. 8, 3159-3165.

[15] _ Semiprime rings with hypercentral derivations, Canad. Math. Bull. 38 (1995), no. 4, 445-449.

[16] , Derivations with Engel conditions on polynomials, Algebra Colloq. 5 (1998), no. $1,13-24$.

[17] T. K. Lee and W. K. Shiue, A result on derivations with Engel condition in prime rings, Southeast Asian Bull. Math. 23 (1999), no. 3, 437-446.

[18] Identities with generalized derivations, Comm. Algebra 29 (2001), no. 10, 44374450.

[19] P. H. Lee and T. L. Wong, Derivations cocentralizing Lie ideals, Bull. Inst. Math. Acad. Sinica 23 (1995), no. 1, 1-5.

[20] W. S. Martindale III, Prime rings satisfying a generalized polynomial identity, J. Algebra 12 (1969), 576-584.

[21] E. C. Posner, Derivations in prime rings, Proc. Amer. Math. Soc. 8 (1957), 1093-1100.

ÇĂ̆RI DEMIR

Department of Mathematics

SCIENCE FACUlTy

EGE UNIVERSITY

35100, Bornova, Izmir, TURKEy

E-mail address: cagri.demir@ege.edu.tr 
Nurcan ArgaÇ

Department of Mathematics

SCIENCE FACULTy

EGE UNIVERSITY

35100, Bornova, Izmir, Turkey

E-mail address: nurcan.argac@ege.edu.tr 